\title{
PARP1 activation promotes FUS translocation to cytoplasm and incorporation into stress granules
}

Anastasia Shavkatovna Singatulina LBCE, Institute of Chemical Biology and Fundamental Medicine SB RAS Novosibirsk, Russia

Bénédicte Desforges

SABNP, INSERM U1204

Université Paris-Saclay Evry, France

\author{
Pastré David \\ SABNP, INSERM U1204 \\ Université Paris-Saclay \\ Evry, France
}

Maria Vladislavovna Sukhanova LBCE, Institute of Chemical Biology and Fundamental Medicin SB RAS Novosibirsk, Russia

\author{
Ahmed Bouhss \\ SABNP, INSERM U1204 \\ Université Paris-Saclay \\ Evry, France \\ Loic Hamon, SABNP \\ INSERM U1204 \\ Université Paris-Saclay \\ Evry, France
}

Olga Ivanovana Lavrik

LBCE, Institute of Chemical Biology and Fundamental Medicine SB RAS

Novosibirsk, Russia

\begin{abstract}
Synthesis of poly(ADP-ribose) catalyzed by nuclear poly(ADP-ribose) polymerases (PARPs) is one of the earliest events in the cellular response to DNA damages in higher eukaryotes. Over the past few years, a huge body of evidence has been accumulated about the involvement of PARPs in the regulation of not only DNA-dependent processes in the nucleus but of RNA-dependent processes in the cell cytoplasm. Many RNA-binding proteins have been described to interact with poly(ADP-ribose) and (or) undergo post-translational modification through poly(ADP-ribosyl)ation. Among them, fused in sarcoma (FUS) protein was found to function in DNA damage response through PARP-dependent mechanism. In the present study, we show that PARP1 activation leads to the shuttling of FUS from the nucleus to the cytoplasm in HeLa cells after hydrogen peroxide -induced oxidative stress. We have observed that stress granule assembly depends on PARP1 activity and found a correlation between the nucleocytoplasmic shuttling of FUS and its accumulation in stress granules, which may in turn promote stress granule assembly. Thus, FUS appears to mediate stress granules formation downstream of PARP1 activation in response to oxidative DNA damage.
\end{abstract}

Keywords - fused in sarcoma, poly(ADP-ribosyl)ation, stress granules

\section{Introduction}

The cellular response to genotoxic stress occurs through a series of regulatory processes that not only control DNA repair but also can cause changes in the level of gene expression [1]. In response to genotoxic impact, DNA damage sensors activate complex signaling networks that affect a number of cellular processes [2]. Nuclear PARPs are sensors of DNA strand breaks and play a key role in the regulation of DNA damage response [3]. Upon binding to DNA breaks, PARPs catalyze the synthesis of poly(ADP-ribose) (PAR) covalently attached to PARPs itself or an acceptor protein using NAD+ as substrate for the post-translation modification [4]. PAR covalently attached to acceptor proteins and can be subsequently hydrolysed to form free mon(ADP-ribose) or oligo(ADP-ribose) by PAR glycohydrolase (PARG) [5]. Recently, PAR has been identified as stress granules (SG) component, and PAR-dependent regulation of nuclearcytoplasmic transport of set of RNA-binding proteins (RBPs) has been shown to involve in the formation of SG [6,7]. SGs are ribonucleoprotein complexes containing untranslated mRNA and RBPs that appear in the cytoplasm of mammalian cells upon the dissociation of polysomes, which occurs generally after various stresses including osmotic stress, oxidative stress, viral infection, hypoxia and hyperthermia [8]. SG dynamics is thought to play a major role in timely mRNArelated processes such as mRNA storage, sorting and protection from degradation. It was shown that PAR produced by nuclear PARPs such PARP1 and PARP2 can function as signaling molecules, driving the translocation of stress granule components from specific subcellular compartments toward the nascent stress granule [9]. In particular, FUS (Fused in Sarcoma) has focused our attention since it is one of the most abundant nuclear RBPs which can be PARylated, interact with PAR and be the component of SGs [10]. Recently, we have found that oxidative stress induces FUS translocation which is dependent on PARP1 and PARG activities [11]. Here, we have analyzed PARP1-dependent formation and composition of stress granules in cells under oxidative stress. We have shown that in hydrogen peroxide -treated cells stress granule assembly was dependent on PARP1 activation despite the absence of PAR in these granules. We have found a correlation between the nucleocytoplasmic shuttling of FUS and its accumulation in stress granules, which may in turn promote stress granule assembly. Thus, the relocation of FUS in the cytoplasm may participate to an adapted translational response to DNA damages but its accumulation in stress granules may be an intermediary step between PARP1 activation and the aggregation of RNA-binding proteins in neurodegenerative diseases.

\section{Results}

To test whether PARP1 activation in nuclear affect the assembly of SGs, we examined the PAR synthesis and SG formation in HeLa cells under oxidative stress induced by arsenite or hydrogen peroxide and puromycin (Fig. 1a). Arsenite is widely used to induce oxidative stress and SGs formation in cells, however, arsenite exposure does not or weakly activates PARP1 in contrast to hydrogen peroxide (Fig. 1a).

In arsenite-treated cells, chemical inhibition of PARP1 by Olaparib does not influence the formation of SGs (Fig. 1b). Nevertheless, SG formation in cells treated with hydrogen peroxide/puromicine is prevented by olaparib (Fig. 1b). We then considered the possibility that PARP1 activation and PAR synthesis could promote stress granule assembly 
no treatment

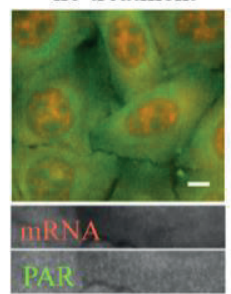

$\mathrm{b}$

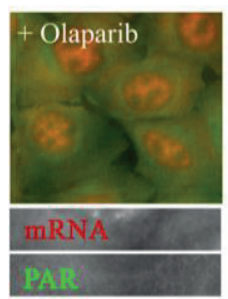

c
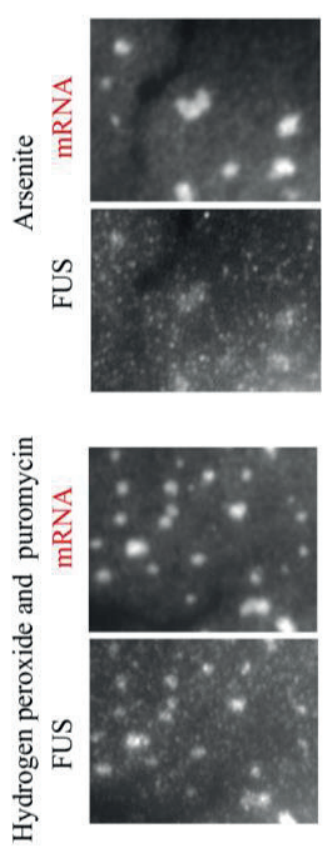

Hydrogen peroxide
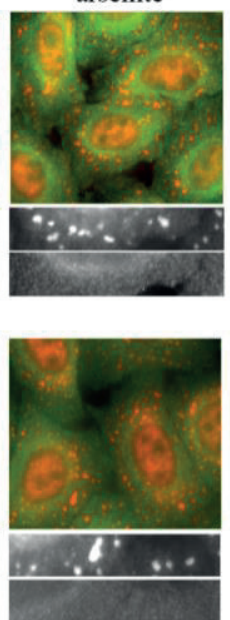

d and puromycin
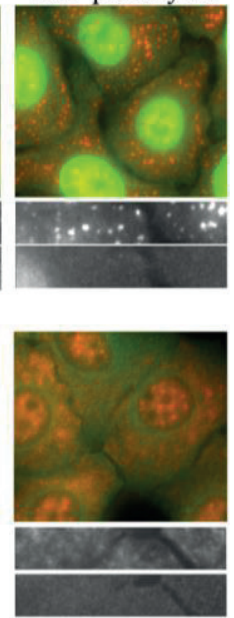

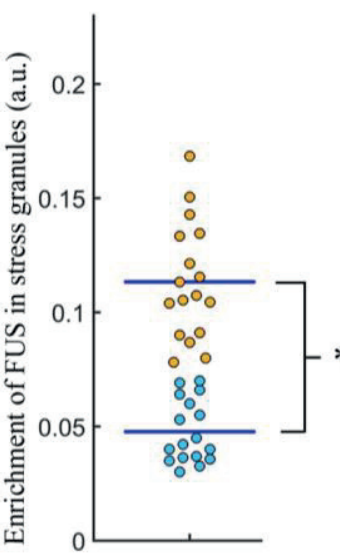

- Arsenite

- Hydrogen peroxide and puromycin

Fig. 1. PARP1 activation is required for the assembly of FUS-rich stress granules after hydrogen peroxide and puromycin treatment. $a$ and $b$. Hela cells were pretreated without (a) or with (b) olaparib for $15 \mathrm{~min}$, then exposed to either arsenite $(300 \mu \mathrm{M}, 60 \mathrm{~min})$ or hydrogen peroxide and puromycin $(300 \mu \mathrm{M}$ and $2.5 \mu \mathrm{g} / \mathrm{mL}$, respectively, 90 min). mRNA was detected by using cy3-labeled poly(T)-probe (red). anti-PAR antibodies fluorescence (green). Scale bar: $10 \mu \mathrm{m}$.

c. Fluorescence images of stress granules in HeLa cells obtained under indicated conditions.

d. Ratio of FUS to mRNA fluorescence integrated intensity in stress granules under indicated conditions. Anti FUS antibodies were used. $\mathrm{p}<0.05, * *$; paired t-test, $\mathrm{n}=17$.

indirectly through the translocation of FUS. To explore this idea, we measured the relative enrichment of FUS in SGs in cells exposed to either arsenite or hydrogen peroxide in combination with puromycin (Fig. 1c and d). We observed

that the accumulation of FUS in SGs is more pronounced in hydrogen peroxide/puromycin - than in arsenite-treated cells (Fig. 1d).

\section{Conclusions}

We hypothesize that FUS could mediate stress granules formation downstream of PARP1 activation in response to oxidative DNA damage. The PARP-dependent relocation of FUS in the cytoplasm may participate in an adapted translational response to DNA damages but FUS accumulation in stress granules may be also an intermediary step between PARP1 activation and the aggregation of RNAbinding proteins such FUS that occurs in neurodegenerative diseases.

\section{ACKNOWLEDGMENT}

The work was supported by RFBR (project no. 18-0400882) and Genopole Evry.

\section{REFERENCES}

[1] M. Dutertre, S. Lambert, A. Carreira, M. Amor-Guéret, and S. Vagner, "DNA damage: RNA-binding proteins protect from near and far", Trends Biochem. Sci., vol. 39, pp. 141-149, March 2014.

[2] L. von Stechow, and J. V. Olsen, "Proteomics insights into DNA damage response and translating this knowledge to clinical strategies", Proteomics, vol. 17, pp. 1600018, December 2017.

[3] M. L. Meyer-Ficca, R.G. Meyer, E.L. Jacobson, and M. K. Jacobson, "Poly (ADP-ribose) polymerases: managing genome stability", Int. J. Biochem. Cell Biol., vol. 37, pp. 920-926, May 2005.

[4] D. d'Amours, S. Desnoyers, I. d'Silva, and G.G. Poirier, "Poly(ADPribosyl)ation reactions in the regulation of nuclear functions", Biochem. J., vol. 342, pp. 249-268, September 1999.

[5] M. E. Bonicalzi, J. F. Haince, A. Droit, and G.G. Poirier, "Regulation of poly(ADP-ribose) metabolism by poly (ADP-ribose) glycohydrolase: where and when?", Cell. Mol. Life Sci., vol. 62, pp. 739-750, April 2005.

[6] A. K. Leung, S. Vyas, J. E. Rood, A. Bhutkar, P. A. Sharp, and P. Chang, "Poly(ADP-ribose) regulates stress responses and microRNA activity in the cytoplasm", Mol. Cell, vol. 42, pp. 489-499, May 2011.

[7] M. Isabelle, J. P. Gagné, I. E. Gallouzi, and G. G. Poirier, "Quantitative proteomics and dynamic imaging reveal that G3BP-mediated stress granule assembly is poly(ADP-ribose)-dependent following exposure to MNNG-induced DNA alkylation", J. Cell Sci., vol. 125, pp. 45554566, October 2012.

[8] J. R. Buchan, and R. Parker, "Eukaryotic stress granules: the ins and outs of translation", Mol. Cell, vol. 36, pp. 932-941, December 2009.

[9] A. K. Leung, "Poly(ADP-ribose): an organizer of cellular architecture", J. Cell Biol., vol. 205, pp. 613-619, June 2014.

[10] R. R. Sama, C. L. Ward, and D. A. Bosco, "Functions of FUS/TLS from DNA repair to stress response: implications for ALS", ASN Neuro, vol. 6, pp. 1759091414544472 , August 2014.

[11] A. S. Singatulina, L. Hamon, M. V. Sukhanova, B. Desforges, V. Joshi, A. Bouhss, O. I. Lavrik, and D. Pastré, "PARP-1 activation directs FUS to DNA damage sites to form PARG-reversible compartments enriched in damaged DNA" Cell Rep., vol. 27, pp. 1809-1821, May 2019. 